\title{
Effect of a standard exercise protocol on shoulder pain in long-term wheelchair users
}

\author{
KA Curtis*,1, TM Tyner ${ }^{1}$, L Zachary ${ }^{1}$, G Lentell $^{1}$, D Brink ${ }^{1}$, T Didyk ${ }^{1}, \mathrm{~K}_{\text {Gean }}{ }^{1}$, J Hall ${ }^{1}$, M Hooper ${ }^{1}, \mathrm{~J} \mathrm{Klos}^{1}$, \\ S Lesina ${ }^{1}$ and B Pacillas ${ }^{1}$ \\ ${ }^{1}$ Department of Physical Therapy, California State University, Fresno, School of Health and Human Services, Fresno, \\ California, USA
}

\begin{abstract}
Purpose: To analyze the effectiveness of a 6-month exercise protocol on shoulder pain experienced by wheelchair users during functional activities.

Subjects: Forty-two wheelchair users, 35 males and seven females: average age of 35 years and an average duration of wheelchair use of 14 years.

Methods: Subjects were randomly assigned to treatment $(n=21)$ and control $(n=21)$ groups. The treatment group received instruction in five shoulder exercises which they performed daily for 6 months. The exercise protocol included two exercises for stretching anterior shoulder musculature and three exercises for strengthening posterior shoulder musculature.

Outcome measures: All subjects completed a self-report questionnaire and the Wheelchair Users Shoulder Pain Index (WUSPI) initially and at bimonthly intervals during the 6-month intervention.

Results: Seventy-five per cent of the subjects reported a history of shoulder pain since beginning wheelchair use. The average initial performance-corrected (PC-WUSPI) score of the 42 subjects was $17.7( \pm 21.3)$ with a range of $0-103.2$ points. Over $83 \%$ of the subjects $(35$ of 42) completed the 6-month study. Subjects in the treatment group decreased their PC-WUSPI score by an average of $39.9 \%$, compared to decreases of only $2.5 \%$ in the control group.

Conclusions: These findings supported the effectiveness of this exercise protocol in decreasing the intensity of shoulder pain which interferes with functional activity in wheelchair users.
\end{abstract}

Keywords: spinal cord injury-treatment; paraplegia; tetraplegia; shoulder; exercise

\section{Introduction}

Early, effective treatment of the individual with spinal cord injury (SCI) has led to an increased life expectancy in this population. With lifetime wheelchair use, often exceeding 20 years, there is an increase in the prevalence of degenerative conditions associated with the aging and overuse of the upper extremities. ${ }^{1-4}$

Published estimates of the prevalence of shoulder pain in individuals with SCI range between $30 \%$ and $100 \%$, indicating that this is a common and persistent problem for individuals with paraplegia or tetraplegia. ${ }^{1-11}$ The high prevalence of shoulder pain seems to be related to increased physical demands and overuse, in that wheelchair users rely exclusively on their arms for their mobility and transfers. ${ }^{3-5}$ Degenerative structural and physiological joint changes related to long-term upper extremity weight-bearing have been reported in subjects with paraplegia. ${ }^{7}$ In addition to chronic degenerative changes, shoulder pain may

*Correspondence: KA Curtis, Physical Therapy Department, California State University, Fresno 2345 E. San Ramon Ave, M/S 29 Fresno, CA 93740-8031, USA develop during the acute rehabilitation period and persist post-discharge. ${ }^{12}$

While shoulder pain may not initially limit the wheelchair user's ability to perform activities independently, it may have functional costs such as rapid fatigue, loss of endurance, decreased speed or efficiency of movement, low tolerance for prolonged work or leisure activity and decreased cardiorespiratory endurance. Eventually wheelchair users with shoulder pain may eliminate functional activities that are associated with pain. ${ }^{8}$

Tightness of the anterior shoulder musculature, combined with weakness of the posterior shoulder musculature both seem to contribute to development of shoulder pain in wheelchair users. ${ }^{13-15}$ Muscle imbalance around the shoulder may be further complicated by paralysis and spasticity in the individual with tetraplegia. 6,14

Strong posterior muscle strength contributes to proper joint function and prevents impingement of soft tissue structures in the sub-acromial space. ${ }^{13,16}$ The rotator cuff muscles exert a downward force which controls upward migration of the humeral head 
created by the pull of the deltoid muscles during humeral elevation. In addition, these muscles control the upward thrust of the humerus during the axial loading of wheelchair propulsion and upper extremity weight bearing activities. ${ }^{13}$

Several authors have proposed interventions to prevent the onset of shoulder pain or to treat chronic shoulder pain in wheelchair users. ${ }^{13,15,17}$ These interventions include increasing the flexibility of the anterior musculature, including the pectoralis and biceps muscles and increasing the muscle strength of the weaker muscles in the posterior shoulder complex, especially muscle groups which control shoulder external rotation, shoulder adduction and scapular retraction. Although these interventions are used in several centers and training programs, efficacy studies have not been published.

The purpose of this study was to analyze the effectiveness of a 6-month exercise protocol on shoulder pain experienced by wheelchair users during the performance of activities of daily living.

\section{Methods}

\section{Subjects}

This pilot study involved a cluster sample of 42 wheelchair users recruited from the community, including 35 men and seven women with an average age of $35( \pm 8)$ years and an average duration of wheelchair use of $14( \pm 9)$ years.

The subjects entered the pilot study in two cohorts, the first consisting of 24 wheelchair users with paraplegia, amputations or neuromuscular disabilities affecting only the lower extremities. The second cohort of 18 subjects included wheelchair users with tetraplegia or upper extremity paralysis.

Subjects reported an average activity level of $26 \mathrm{~h}$ of work, $12 \mathrm{~h}$ of leisure and sporting activity, and $8 \mathrm{~h}$ of driving per week. They reported an average of $11( \pm 10)$ wheelchair transfers per day. Seventyeight per cent of the subjects were employed or fulltime students. Subject disabilities included SCI $(n=35)$, amputation $(n=2)$, cerebral palsy $(n=2)$, post-polio paralysis $(n=2)$ and multiple sclerosis $(n=1)$.

All subjects used a manual wheelchair at least $3 \mathrm{~h}$ per week and had a neurological level (motor and sensory levels) at C-6 or lower, using American Spinal Injury Association (ASIA) guidelines. All subjects were at least 1 year post-onset of their disabilities. We excluded subjects that had experienced upper extremity fractures, dislocations or surgery during the study or within 6 months prior to their participation in the study. In addition, subjects were excluded if they experienced active medical problems which required hospitalization within 1 month of the initiation of the study or at any time during the study.

\section{Instrumentation}

A self-report questionnaire was used to collect demographic and medical history information and to assess the intensity of pain experienced during the performance activities of daily living. Collected data included age, gender, marital status, onset and type of disability, duration of wheelchair use, level of neurological involvement, frequency of wheelchair transfers per day, hours per week spent at work and/ or school, sport and leisure activities and driving, type of automobile driven, history of shoulder problems, presence and location of current shoulder pain, presence of other upper extremity pain, health care providers consulted and treatment received for shoulder pain.

We used the Wheelchair User's Shoulder Pain Index (WUSPI), a 15-item valid and reliable self-report questionnaire to assess the intensity of pain during the performance of activities of daily living such as transfers, loading a wheelchair into a car, wheeling up inclines, dressing, bathing, overhead lifting, driving, performing household chores, and sleeping. ${ }^{8,18}$ Subjects rated their intensity of pain during these activities on a $10 \mathrm{~cm}$ visual analog scale (VAS) anchored at 'no pain' and 'worst pain ever experienced.' The total possible score on the scale ranged from 0 to 150 .

When tested for reliability, the WUSPI showed high internal consistency $(\alpha=0.97)$ and high test-retest reliability $(\mathrm{ICC}=0.99){ }^{8,18}$ In analysis of concurrent validity, the WUSPI showed significant negative correlations with shoulder range of motion measurements of abduction $(r=-0.49)$, flexion $(r=-0.48)$ and shoulder extension $(r=-0.30) .{ }^{18}$

\section{Procedures}

The subjects were screened to insure that they met study eligibility criteria. Subjects signed a consent form, in accordance with procedures approved by the Institutional Review Board for the Protection of Human Subjects. All subjects completed initial questionnaires and were then randomly assigned to a control group $(n=21)$ or treatment group $(n=21)$. Both groups were instructed to continue their daily activities as usual throughout the duration of the study. All subjects in both the treatment and control groups completed the WUSPI at the initiation of the study and every 2 months thereafter for a 6 month period.

\section{Intervention}

The subjects in the treatment group attended a $60 \mathrm{~min}$ educational session where they were instructed in five daily shoulder exercises. The exercise program included two static stretching exercises to increase the flexibility of the anterior shoulder musculature and three resistive strengthening exercises for the posterior shoulder muscles (see Appendix). Subjects performed the exercises indepen- 
dently in their wheelchairs using lightweight stretchable exercise bands for resistance. Subjects with tetraplegia used a webbing wrist strap, when necessary, to accommodate for weak or absent grasp. All subjects received all exercise materials and a written summary for reference at home.

The instructional session included an introduction to functional shoulder anatomy, purpose of the

Table 1 Comparisons of subjects in control and treatment groups on age, exposure and activity characteristics by unpaired $t$-tests

\begin{tabular}{|c|c|c|c|c|c|}
\hline & \multicolumn{2}{|c|}{ Control group $(\mathrm{n}=21)$} & \multicolumn{2}{|c|}{ Treatment group $(\mathrm{n}=21)$} & \multirow[b]{2}{*}{$\mathrm{P}$ value } \\
\hline & Mean & $S D$ & Mean & $S D$ & \\
\hline Age & 35.7 & 9.0 & 34.5 & 6.7 & NS \\
\hline Years of wheelchair use & 13.1 & 10.4 & 14.9 & 8.0 & NS \\
\hline Average number of wheelchair transfers/day & 9.3 & 10.8 & 13.7 & 9.1 & NS \\
\hline Hours/week of work and school & 27.6 & 19.8 & 24.0 & 18.0 & NS \\
\hline Hours/week of sport and leisure & 12.0 & 10.1 & 11.8 & 9.3 & NS \\
\hline Hours/week of driving & 9.0 & 7.3 & 7.4 & 5.8 & NS \\
\hline
\end{tabular}

NS: indicates that $\mathrm{P}>0.05$

Table 2 Comparisons of subjects in control and treatment groups by Chi-square analyses on demographic characteristics

\begin{tabular}{|c|c|c|c|c|c|}
\hline & \multicolumn{2}{|c|}{ Control group $(\mathrm{n}=21)$} & \multicolumn{2}{|c|}{ Treatment group $(\mathrm{n}=21)$} & $\mathrm{P}$ value \\
\hline Gender & & & & & NS \\
\hline Male & 17 & 81.0 & 18 & 85.7 & \\
\hline Female & 4 & 19.1 & 3 & 14.3 & \\
\hline Marital status & & & & & NS \\
\hline Single & 11 & 52.4 & 7 & 33.3 & \\
\hline Married & 8 & 38.1 & 11 & 52.4 & \\
\hline Divorced & 1 & 4.8 & 3 & 14.3 & \\
\hline Separated & 1 & 4.8 & 0 & 0 & \\
\hline Widowed & 0 & 0.0 & 0 & 0 & \\
\hline Wheelchair type & & & & & NS \\
\hline Manual only & 19 & 90.5 & 18 & 85.7 & \\
\hline Both manual and power & 2 & 9.5 & 3 & 14.3 & \\
\hline Disability & & & & & NS \\
\hline Spinal cord injury & 16 & 76.2 & 19 & 90.5 & \\
\hline Post-polio paralysis & 1 & 4.8 & 1 & 4.8 & \\
\hline Amputation & 2 & 9.5 & 0 & 0 & \\
\hline Cerebral palsy & 2 & 9.5 & 0 & 0 & \\
\hline Multiple sclerosis & 0 & 9.5 & 1 & 4.8 & \\
\hline Neurological lesion level & & & & & NS \\
\hline Cervical & 8 & 43.8 & 10 & 50.0 & \\
\hline Thoracic & 8 & 50.0 & 10 & 50.0 & \\
\hline Lumbar & 1 & 6.2 & 0 & 0 & \\
\hline Employment status & & & & & NS \\
\hline Employed & 10 & 47.6 & 12 & 57.2 & \\
\hline Student & 6 & 28.6 & 5 & 23.8 & \\
\hline Volunteer & 1 & 4.8 & 1 & 4.8 & \\
\hline Retired & 2 & 9.5 & 0 & 0 & \\
\hline Other & 2 & 9.5 & 3 & 14.3 & \\
\hline Driving & & & & & NS \\
\hline Yes & 16 & 76.2 & 17 & 81.0 & \\
\hline No & 5 & 23.81 & 4 & 19.1 & \\
\hline Vehicle type & & & & & NS \\
\hline Car & 8 & 50 & 8 & 53.3 & \\
\hline Van with lift & 2 & 12.5 & 3 & 20 & \\
\hline Van without lift & 2 & 12.5 & 0 & 0 & \\
\hline Truck or utility vehicle & 4 & 25 & 4 & 26.7 & \\
\hline
\end{tabular}

NS: Indicates that $P>0.05$ 
exercises, demonstration, and individual instruction on how to perform the exercises. Research assistants checked for correct performance of each exercise and gave each subject feedback with correction as necessary.

We instructed treatment group subjects to perform the stretching exercises twice daily, five times each, with a 20-30 s hold of each position to promote a prolonged muscle stretch and to perform the strengthening exercises once daily and complete three sets of 15 repetitions. We emphasized proper body mechanics to ensure correct execution of the stretching and strengthening exercises. Throughout the course of the study, subjects in the treatment group kept a $\log$ recording daily performance of exercises and any shoulder injuries or accidents which occurred.

Performance and problems were monitored by biweekly telephone calls between data collection intervals. Performance was reviewed, corrected, if necessary and reinforced at the 2 and 4-month data collection points.

\section{Data analysis}

All demographic data were analyzed using descriptive statistics. The age, years of wheelchair use and activity levels of subjects in the control and treatment groups were compared by independent $t$-tests. We compared medical history characteristics and prevalence of shoulder pain in the two groups by Chi-square analysis.

The WUSPI was scored by measuring the response on the visual analog scales for each of the 15 items. The individual item scores were then summed to give a total score. We recorded how many subjects marked the option of 'not performed' instead of a score on the visual analog scale. Because some of the subjects with tetraplegia did not perform one or more activities measured in WUSPI items, we calculated a performance-corrected (PC-WUSPI) score for all subjects by dividing the raw total WUSPI score by the number of activities performed and then multiplying by 15 . In this way, we were able to more accurately reflect the actual intensity of shoulder pain experienced during activities performed, rather than assuming equivalent activity levels in subjects with paraplegia and tetraplegia.

A repeated measures analysis of variance to compare the effect of the exercise intervention on the subject's shoulder pain (PC-WUSPI) scores over the duration of the study was carried out.

\section{Results}

At the initiation of the study, unpaired $t$-tests showed no significant differences between the control and treatment group subjects in age, years of wheelchair

Table 3 Comparisons of subjects with control and treatment groups by Chi-square analyses on history of shoulder pain and treatment at the initiation of the study

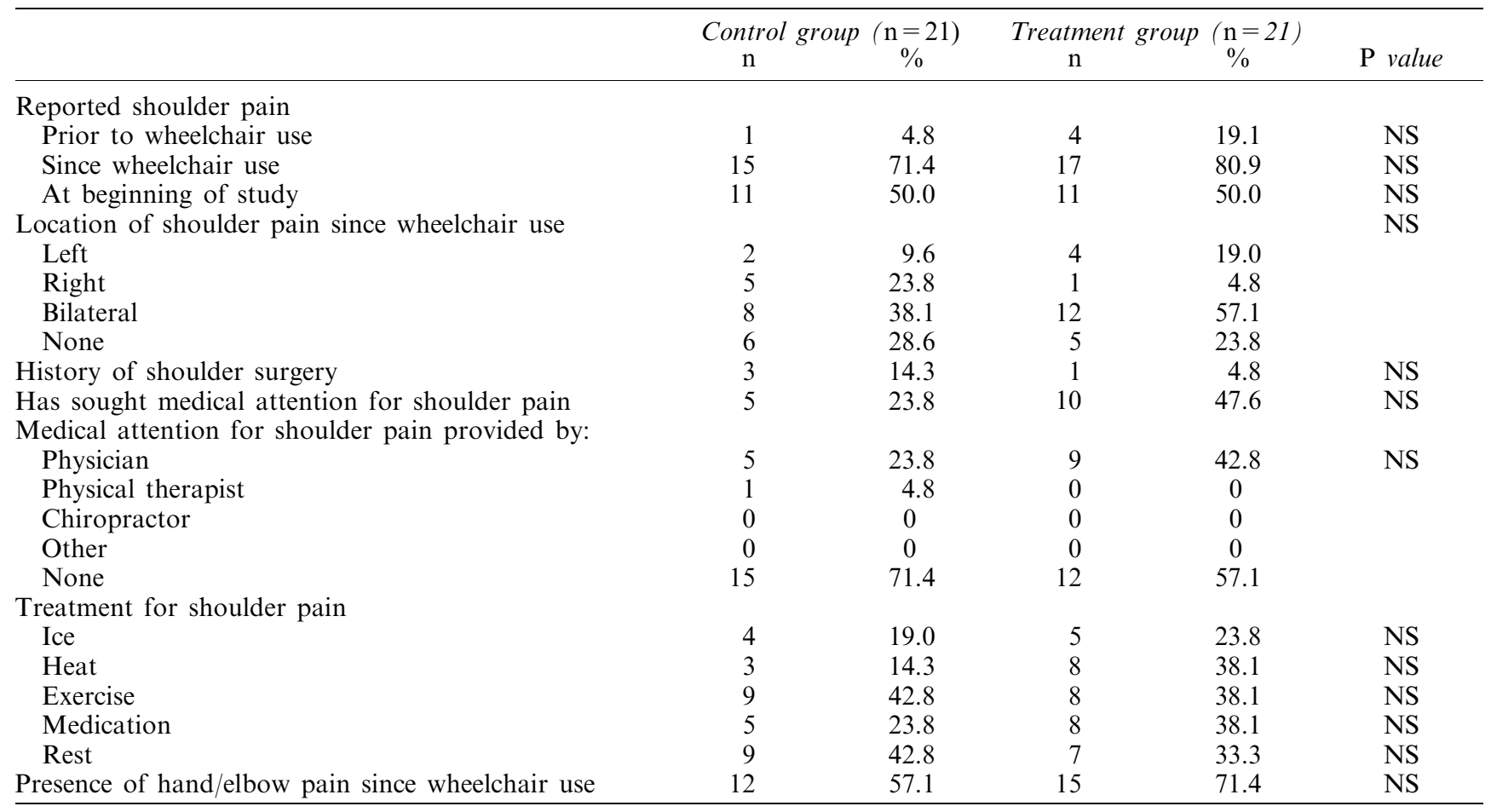

NS: Indicates that $P>0.05$ 
use or activity levels (Table 1). In addition, Chi-square analyses showed no significant differences between the control and treatment group subjects in gender, marital status, type of disability, level of spinal cord injury status, wheelchair, driving status and type of vehicle (Table 2).

Over $75 \%$ of all subjects reported a history of shoulder pain and $64 \%$ reported hand or elbow pain since beginning wheelchair use. At initiation of the study, $50 \%$ of both the control and treatment group subjects reported current shoulder pain. Chi-square analyses of upper extremity pain and treatment history characteristics showed no significant differences between the control and treatment group subjects (Table 3).

Over $80 \%$ (35 of the 42 subjects) completed the 6-month intervention. Overall, two subjects left the study due to medical conditions and five subjects left the study due to time constraints, leaving 18 subjects in the control group and 17 subjects in the treatment group at the completion of the study. Three treatment group subjects sustained sportsrelated shoulder injuries while enrolled in the study and one of these subjects did not perform the exercise intervention for the last 3 months of the study.

The average initial PC-WUSPI score of the 42 subjects was $17.7( \pm 21.3)$ with a range of $0-103.2$ points. The change in mean PC-WUSPI scores over the 6-month intervention was calculated for the treatment and control groups (Table 4). At the end of the intervention, the subjects in the treatment group had decreased their mean PC-WUSPI score by 9.3 points $(39.3 \%)$, compared to a decrease of 0.3 points $(2.5 \%)$ in the control group (Figure 1).

The ten subjects with paraplegia in the treatment group showed a $48.3 \%$ decrease in their WUSPI scores, where seven subjects with tetraplegia in the treatment group showed a $27.2 \%$ decrease in shoulder

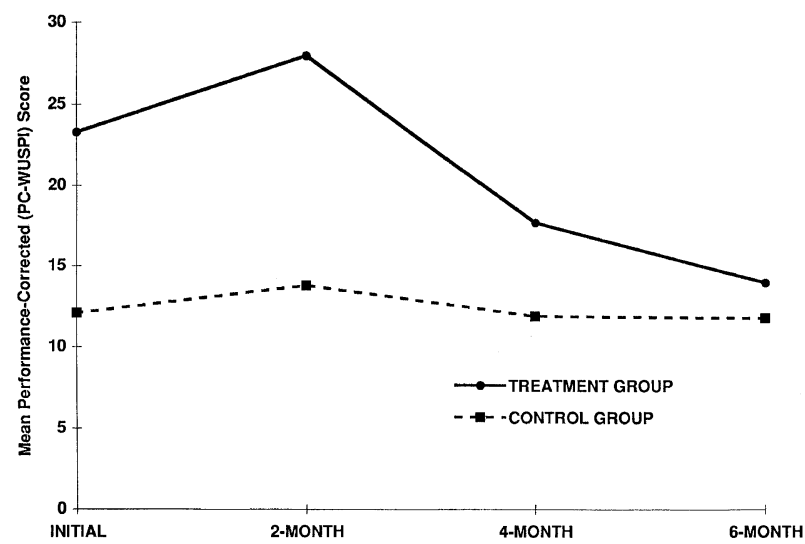

Figure 1 Subjects in the treatment group decreased their performance-corrected Wheelchair User Shoulder Pain Index (PC-WUSPI) scores by 9.3 points, compared to 0.3 points in the control group pain. The eleven subjects with paraplegia in the control group showed a $18.1 \%$ decrease in their shoulder pain, and the seven subjects with tetraplegia in the control group showed a $8.8 \%$ increase in their shoulder pain (Figures 2 and 3).

Analysis of the effect of the exercise intervention on PC-WUSPI score, using a two-factor repeated measures ANOVA, showed a significant effect of time $(P=0.048)$ but failed to show a significant effect of treatment or an interaction between time and treatment (Table 5).

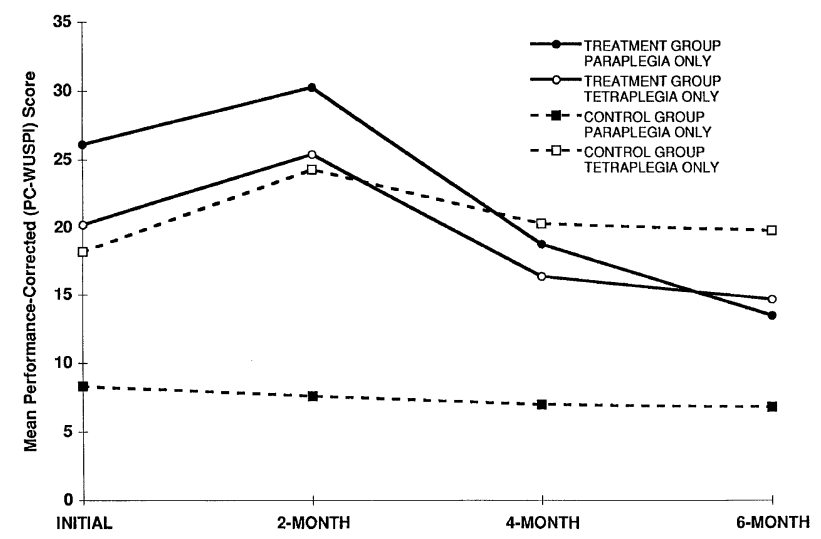

Figure 2 Ten subjects with paraplegia in the treatment group experienced a 12.1 point decrease in their performancecorrected Wheelchair User Shoulder Pain Index (PC-WUSPI) scores compared to a 1.5 point drop in 11 control group subjects with paraplegia. The seven control group subjects with tetraplegia reported a 5.5 point score decrease, compared with seven subjects with tetraplegia in the control group subjects who had 1.6 point score increase

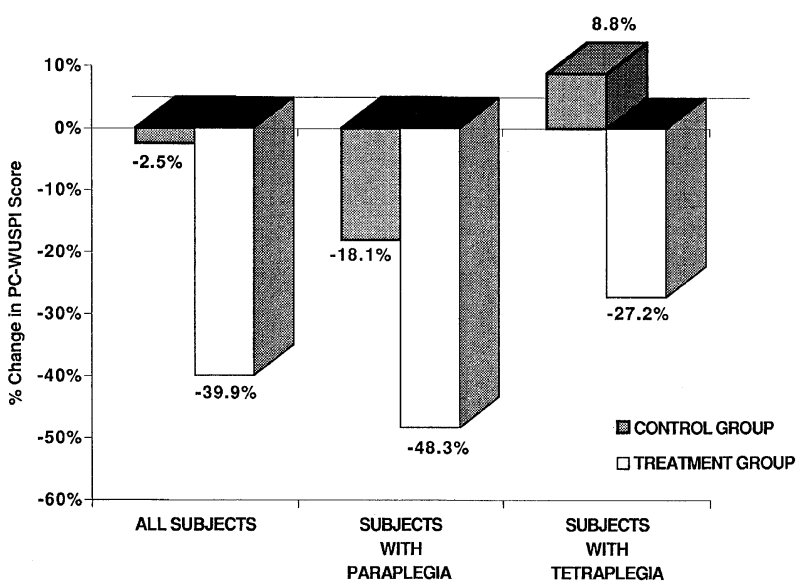

Figure 3 The intervention appears to have been maximally effective for ten treatment group subjects with paraplegia, reflecting a $48.3 \%$ decrease in their performance-corrected Wheelchair User Shoulder Pain Index (PC-WUSPI) scores over the 6-month intervention 
Table 4 Initial and 6-month Wheelchair User's Shoulder Pain Index (WUSPI) scores in control and treatment groups

\begin{tabular}{|c|c|c|c|c|c|c|c|c|}
\hline \multirow[b]{3}{*}{ WUSPI items } & \multicolumn{4}{|c|}{ Initial scores } & \multicolumn{4}{|c|}{6 month scores } \\
\hline & \multicolumn{2}{|c|}{$\begin{array}{l}\text { Control group } \\
(\mathrm{n}=21)\end{array}$} & \multicolumn{2}{|c|}{$\begin{array}{l}\text { Experimental group } \\
\qquad(\mathrm{n}=21)\end{array}$} & \multicolumn{2}{|c|}{$\begin{array}{l}\text { Control group } \\
(\mathrm{n}=18)\end{array}$} & \multicolumn{2}{|c|}{$\begin{array}{l}\text { Experimental group } \\
\qquad(\mathrm{n}=17)\end{array}$} \\
\hline & Mean & $S D$ & Mean & $S D$ & Mean & $S D$ & Mean & $S D$ \\
\hline Bed-wheelchair transfer & 0.6 & 1.1 & 1.3 & 2.5 & 1.0 & 1.6 & 0.8 & 1.3 \\
\hline Wheelchair-car transfer & 0.3 & 0.8 & 1.4 & 1.8 & 1.1 & 2.2 & 1.2 & 1.7 \\
\hline Wheelchair-tub transfer & 0.4 & 0.7 & 1.2 & 1.9 & 1.0 & 2.0 & 1.1 & 1.6 \\
\hline Loading wheelchair into car & 1.0 & 1.5 & 1.7 & 2.2 & 0.8 & 1.6 & 0.9 & 1.6 \\
\hline Pushing wheelchair > $10 \mathrm{~min}$ & 1.4 & 1.8 & 2.2 & 2.4 & 1.0 & 1.6 & 0.9 & 1.3 \\
\hline Pushing wheelchair up ramps/inclines & 1.3 & 1.9 & 2.2 & 2.5 & 1.0 & 1.7 & 1.2 & 1.6 \\
\hline Lifting object from overhead & 1.5 & 2.2 & 1.8 & 2.4 & 0.9 & 1.4 & 1.6 & 2.2 \\
\hline Putting on pants & 0.4 & 0.7 & 1.0 & 2.3 & 0.5 & 1.1 & 1.0 & 1.7 \\
\hline Putting on t-shirt/pullover & 0.3 & 0.8 & 1.1 & 2.1 & 0.3 & 0.6 & 0.5 & 1.1 \\
\hline Putting on button down shirt & 0.2 & 0.6 & 1.1 & 2.2 & 0.2 & 0.4 & 0.5 & 1.0 \\
\hline Washing back & 0.5 & 1.1 & 1.3 & 2.4 & 0.3 & 0.5 & 0.8 & 1.4 \\
\hline Daily activities at work/school & 1.0 & 1.7 & 1.6 & 2.1 & 0.9 & 1.5 & 1.4 & 1.9 \\
\hline Driving & 0.9 & 1.6 & 1.3 & 1.4 & 0.4 & 1.0 & 0.5 & 1.1 \\
\hline Household chores & 1.2 & 2.3 & 1.7 & 2.8 & 0.8 & 1.6 & 0.7 & 1.3 \\
\hline Sleeping & 1.6 & 2.4 & 2.2 & 3.1 & 1.2 & 2.3 & 1.5 & 2.0 \\
\hline $\begin{array}{l}\text { Raw WUSPI score } \\
\text { (prior to performance correction) }\end{array}$ & 11.5 & 13.0 & 21.5 & 24.3 & 11.1 & 17.1 & 13.2 & 19.1 \\
\hline $\begin{array}{l}\text { Performance-corrected Wheelchair User's } \\
\text { Shoulder Pain Index (PC-WUSPI) } \\
\text { Score }\end{array}$ & 12.1 & 13.3 & 23.3 & 26.2 & 11.8 & 17.6 & 14.0 & 20.1 \\
\hline
\end{tabular}

Table 5 Repeated measures of analysis of variance (ANOVA) table analyzing the effect of the treatment on PerformanceCorrected Wheelchair User Shoulder Pain Index (PC-WUSPI) scores

\begin{tabular}{|c|c|c|c|c|c|}
\hline Source & $d f$ & Sum of squares & Mean square & F-test & $\mathrm{P}$ value \\
\hline (A) Treatment & 1 & 1132.7 & 1132.7 & 0.8 & 0.3866 \\
\hline Subjects w. groups & 33 & 48558.7 & 1471.5 & & \\
\hline (B) (Time) Repeated measure PC-WUSPI Score & 3 & 1035.5 & 345.2 & 2.7 & 0.0481 \\
\hline $\mathrm{AB}$ interaction & 3 & 394.4 & 131.5 & 1.0 & 0.379 \\
\hline $\mathrm{B} \times$ subjects $\mathrm{w}$. groups & 99 & 12529.7 & 126.6 & & \\
\hline
\end{tabular}

\section{Discussion}

Shoulder pain is a common and persistent problem among wheelchair users. Wheelchair users rely exclusively on their upper extremities for their mobility and transfers. Muscle imbalance, postural changes, chronic overuse and repetitive trauma have all been implicated in the pathogenesis of shoulder problems in this population. $^{13,16,17}$

This may be attributed in part to the repetitive nature of the stroke pattern involved in wheelchair propulsion. ${ }^{13,15,17}$ This repetitive movement encourages protraction and elevation of the scapula with relative internal rotation of the humerus. Pain often results as the soft tissues of the rotator cuff become impinged in the narrowed sub-acromial space during humeral elevation, especially with abduction and internal rotation. ${ }^{13,16,17}$ Weight-bearing during transfers and ambulation may add further stress. ${ }^{7}$
This intervention addressed the above postural changes and related muscular imbalance, using the rationale that weak posterior shoulder musculature is unlikely to provide sufficient shoulder stability to promote normal upper torso posture, normal shoulder joint movement or to prevent impingement in the sub-acromial space. Several authors have suggested posterior shoulder muscle strengthening to prevent this problem. ${ }^{13,15}$ Subjects in this study performed strengthening exercises for scapular retraction with shoulder horizontal abduction, shoulder external rotation and shoulder adduction.

Posterior weakness may be further exacerbated by anterior shoulder muscle shortening, promoting upper torso postural changes characterized by upper thoracic kyphosis, scapular protraction and elevation and internal rotation of the humerus. Subjects in this study performed two stretching exercises to address these postural changes. 


\section{Limitations of the study}

Standardized treatment protocol This study, by design, tested the effect of a standardized intervention, rather than the effect of an individually-prescribed, problem-specific shoulder treatment program. We recognize that individual subjects may have had problems which involved degenerative joint changes, inflammation of the joint capsule or ligamentous laxity about the shoulder. In these cases, one could argue that there would be more appropriate treatment interventions.

We would expect an equal distribution of subjects with similar problems in each of the randomly assigned treatment and control groups. We did not perform individual evaluations on each subject and have no radiological findings or other diagnostic data to confirm or disconfirm this assumption.

Differences in subject neurological function The findings of this study show a decrease in PC-WUSPI scores in ten treatment group subjects with paraplegia $(-48.3 \%)$ which is almost twice as great as that seen in seven treatment group subjects with tetraplegia $(-27.2 \%)$. These findings may reflect differences in neurological function, specifically in innervation of muscle groups from the $\mathrm{C} 7$ and $\mathrm{C} 8$ myotomes. Although all subjects with tetraplegia had C-6 muscle function they may have differed in the strength of muscles partially innervated by the C-7 and C-8 levels. We did not test our subjects to monitor the strength of each of the targeted muscle groups. It may be interesting in a larger sample to specifically analyze these possible trends.

In addition, subjects with paraplegia and subjects with tetraplegia entered the study as two different cohorts. Although we kept the intervention consistent between the two cohorts, history and environmental factors may influence these results.

Longitudinal nature of the problem and solution In longitudinal research designs, subject attrition and noncompliance becomes a serious problem with increasing length of studies. We were able to retain over $80 \%$ of our subjects in this 6-month intervention.

However, the evolution of shoulder problems in wheelchair users is a long-term process and solutions to counteract such a problem may take in excess of 6 months. Thus, the 6-month termination of this study may have missed a continuing downward trend in shoulder pain scores.

In addition, this intervention appears to have a variable effect in the initial 2 months, where shoulder pain scores increased in the treatment group before decreasing at the 4 and 6-month measurement points. The documentation of an initial increase in pain scores is an important finding, as it may deter health professionals who are measuring treatment outcomes in this shorter-time interval from fully realizing the effectiveness of a long term intervention of this nature.
Self-treatment and compliance issues Since this was a home exercise program, subjects did not have the benefit of professional personnel to oversee their regular performance of the program or to correct ineffective or incorrect performance. Our findings also reflect real-life constraints of motivation, time pressures and priorities in the lives of our subjects. The effect we saw in this largely self-administered intervention lends support to similar types of patient education efforts.

\section{Clinical implications}

Health care providers are forced to look for solutions which not only alleviate but prevent common problems at a minimal cost. This shoulder care program seems to be such a solution.

We propose that a simple shoulder exercise program, such as the one outlined in this study, becomes standard instructions for the prevention of shoulder pain in wheelchair users. Such instruction could easily be incorporated with other prevention instructions such as weight-shifting to prevent the development of decubitus ulcers or performing self range of motion exercises to prevent joint contracture.

\section{Needs for future study}

Wheelchair users experience numerous musculoskeletal problems with aging. Sitting posture, wheelchair push stroke, activity and fitness levels may all be related to the development of musculoskeletal problems and provide avenues for future research in this population.

In addition, the effect of a prevention program introduced at the initiation of wheelchair use may have value in delaying the onset of shoulder problems or minimizing the functional consequences of shoulder pain in this population. The incidence, intensity and duration of shoulder problems needs to be documented in relation to long-term prevention efforts. Finally, inclusion of more subjects in a similar longitudinal design would dampen the effect of a high intra-group variation on statistical power.

\section{Acknowledgements}

This study was supported by grants from the Department of Health and Human Services, California State University, Fresno and the California Physical Therapy Fund, Inc.

\section{References}

1 Gellman H, Sie I, Waters RL. Late complications of the weightbearing extremity in the paraplegic patient. Clin Orthopaed Relat Res 1988; 233: $132-135$.

2 Sie IH, Waters RL, Adkins RH, Gellman H. Upper extremity pain in the post-rehabilitation spinal cord injured patient. Arch Phys Med Rehabil 1992; 73(1): 44-48.

3 Pentland WE, Twomey LT. The weight-bearing upper extremity in women with long term paraplegia. Paraplegia 1991; 29: 521 - 530. 
4 Pentland WE, Twomey LT. Upper limb function in persons with long term paraplegia and implications for independence: part I \& II. Paraplegia 1994; 32: $211-224$.

5 Nichols PJR, Norman PA, Ennis JR. Wheelchair user's shoulder? Shoulder pain in patients with spinal cord lesions. Scand J Rehab Med 1979; 11: 29-32.

6 Silfverskiold J, Waters RL. Shoulder pain and functional disability in spinal cord injury patients. Clin Orthopaed Relat Res 1991; 272: 141 - 145.

7 Bayley JC, Cochran PC, Sledge CB. The weight bearing shoulder: the impingement syndrome in paraplegics. J Bone J Joint Surg 1987; 69: 676-678.

8 Curtis KA et al. Development of the wheelchair user's shoulder pain index (WUSPI). Paraplegia 1995; 33: 290-293.

9 MacKay-Lyons M. Shoulder pain in patients with acute quadriplegia: a retrospective study. Physiotherapy Canada 1994; 46: $255-258$.

10 Campbell CC, Koris MJ. Etiologies of shoulder pain in cervical spinal cord injury. Clin Orthopaed Relat Res 1996; 322: 140-145.

11 Waring WP, Maynard FM. Shoulder pain in acute traumatic quadriplegia. Paraplegia 1991; 29: $37-42$.

12 Scott JA, Donovan WH. The prevention of shoulder pain and contracture in the acute tetraplegia patient. Paraplegia 1981; 19: $313-319$.

13 Burnham RS, et al. Shoulder pain in wheelchair athletes: The role of muscle imbalance. Am J Sports Med 1993; 21(2): 238-242.

14 Powers CM et al. Isometric shoulder torque in subjects with spinal cord injury. Arch Phys Med Rehabil 1994; 75: 761 - 765.

15 Millikan T, Morse M, Hedrick B. Prevention of shoulder injuries. Sports 'n Spokes 1991; 17: 35-38.

16 Apple DF, Cody R, Allen A. Overuse syndrome of the upper limb in people with spinal cord injury. In: Apple DF (ed). Physical Fitness: A Guide for Individuals with Spinal Cord Injury. Department of Veterans Affairs, Rehabilitation Research and Development Service, Scientific and Technical Publications Section, Washington D.C.

17 Burnham RS, Curtis KA, Reid DC. Shoulder problems in the wheelchair athlete. In: Petrone FA (ed). Athletic Injuries of the Shoulder. McGraw-Hill, Inc.: New York.

18 Curtis KA et al. Reliability and validity of the wheelchair user's shoulder pain index (WUSPI). Paraplegia 1995; 33: 595-601.

\section{Appendix}

\section{Description of exercise protocol}

Exercises are to be performed daily, 7 days per week.

\section{Strengthening Exercises}

All strengthening exercises are done one arm at a time with strap secured firmly around wrist while other end is attached to a doorknob or a similar stable object. Position wheelchair far enough away to take up the slack in the band.

You may feel fatigued while performing these exercises but you should not feel a sharp pain. If you feel a sharp pain, STOP and call the research team.

\section{Scapular retraction}

- Attach band to wrist.

- Face the door so that the band is in front of you with your arm straight.

- With arm at chest level, pull one arm back in a rowing motion.
- Return slowly to the starting position.

- Repeat for 3 sets of 15 repetitions.

- Repeat with other arm.

\section{Shoulder External Rotation}

- Turn wheelchair so that you are at a $90^{\circ}$ angle to the door.

- Attach the band to the wrist that is furthest from the door.

- Place yourself far enough away so that the band's tension allows your hand to rest on your abdomen, with your elbow bent to a $90^{\circ}$ angle, with your elbow at your side.

- Keeping your elbow at your side and bent $90^{\circ}$, pull your wrist away from the door and bring your wrist as far as you can toward the opposite side.

- Your elbow should stay at your side and bent throughout the motion.

- Return slowly to the starting position.

- Repeat for 3 sets of 15 repetitions.

- Repeat with other arm.

\section{Shoulder Adduction}

- Position your wheelchair so that you are at a $90^{\circ}$ angle to the door with your elbow straight.

- Attach the band to the wrist that is closest to the door.

- Pull your wrist down toward your side while keeping your elbow straight and thumb pointed down.

- Return slowly to the starting position.

- Repeat for 3 sets of 15 repetitions.

- Repeat with other arm.

\section{Stretching Exercises:}

All stretching exercises are done with wheelchair positioned in doorway so that the arm to be stretched will just fit behind the door jamb without having to lean forward or backward in the wheelchair.

You should feel some tension in the muscle with a firm prolonged stretch, but never a sharp pain. If you feel a sharp pain, STOP and call the research team.

\section{Pectoral Stretch}

- With elbow at shoulder height and elbow bent to $90^{\circ}$, place forearm on doorjamb, making contact from elbow to wrist.

- Lean slightly forward until a firm stretch is felt in the chest region.

- Repeat for 5 repetitions, holding for 20 to 30 seconds each time.

- Repeat with the other arm.

\section{Biceps Stretch}

- Position arm at a $45^{\circ}$ angle, elbow straight and thumb up. 
- Place lower forearm against doorjamb. You may hold onto the doorjamb if you would like.

- Rotate wheelchair AWAY from arm until you feel a stretch in the biceps muscle.
- Repeat for 5 repetitions, holding for 20 to 30 seconds each time.

- Repeat with the other arm. 